\title{
Effects of Transcranial Direct Current Stimulation over Left Dorsolateral pFC on the Attentional Blink Depend on Individual Baseline Performance
}

ARTICLE in JOURNAL OF COGNITIVE NEUROSCIENCE · AUGUST 2015

Impact Factor: 4.09 · DOI: 10.1162/jocn_a_00867 · Source: PubMed

2 AUTHORS:

Raquel London

Ghent University

3 PUBLICATIONS 43 CITATIONS

SEE PROFILE
Heleen Slagter

University of Amsterdam

51 PUBLICATIONS 2,938 CITATIONS

SEE PROFILE 


\title{
Effects of Transcranial Direct Current Stimulation over Left Dorsolateral pFC on the Attentional Blink Depend on Individual Baseline Performance
}

\author{
Raquel E. London ${ }^{1}$ and Heleen A. Slagter ${ }^{2}$
}

\begin{abstract}
Selection mechanisms that dynamically gate only relevant perceptual information for further processing and sustained representation in working memory are critical for goal-directed behavior. We examined whether this gating process can be modulated by anodal transcranial direct current stimulation (tDCS) over left dorsolateral pFC (DLPFC) - a region known to play a key role in working memory and conscious access. Specifically, we examined the effects of tDCS on the magnitude of the so-called "attentional blink" (AB), a deficit in identifying the second of two targets presented in rapid succession. Thirtyfour participants performed a standard $\mathrm{AB}$ task before (baseline), during, and after $20 \mathrm{~min}$ of $1-\mathrm{mA}$ anodal and cathodal tDCS in two separate sessions. On the basis of previous reports linking individual differences in $\mathrm{AB}$ magnitude to individual differences in DLPFC activity and on suggestions that effects of tDCS depend on baseline brain activity levels, we hypothesized
\end{abstract}

\section{INTRODUCTION}

Our senses constantly convey a barrage of information to our brain, overwhelming its limited processing capacity. One phenomenon that brings this fact to light is the attentional blink $(\mathrm{AB})$. When given the task to detect two targets among a stream of distractors presented in rapid succession, participants are often unable to identify the second target (T2) if it is presented within approximately 200-500 msec of the first target (T1; Raymond, Shapiro, \& Arnell, 1992). It is as if attention temporarily "blinks." Since its discovery, a variety of models of the AB have been proposed that differ considerably in their specific architectures (Martens \& Wyble, 2010; Dux \& Marois, 2009; Olivers \& Meeter, 2008; Shapiro, Raymond, \& Arnell, 1997). Nevertheless, working memory (WM) plays a central role in virtually all of them. Initially, many theories relied on the idea that the $\mathrm{AB}$ reflects a limitation in processing resources required for the encoding and consolidation of target information in WM. For example, it was postulated that, when many WM resources are devoted to the processing of T1, too few may be available

${ }^{1}$ Ghent University, ${ }^{2}$ University of Amsterdam that anodal tDCS over left DLPFC would modulate the magnitude of the $\mathrm{AB}$ as a function of individual baseline $\mathrm{AB}$ magnitude. Indeed, individual differences' analyses revealed that anodal tDCS decreased the AB in participants with a large baseline $\mathrm{AB}$ but increased the $\mathrm{AB}$ in participants with a small baseline $\mathrm{AB}$. This effect was only observed during (but not after) stimulation, was not found for cathodal tDCS, and could not be explained by regression to the mean. Notably, the effects of tDCS were not apparent at the group level, highlighting the importance of taking individual variability in performance into account when evaluating the effectiveness of tDCS. These findings support the idea that left DLPFC plays a critical role in the $\mathrm{AB}$ and in conscious access more generally. They are also in line with the notion that there is an optimal level of prefrontal activity for cognitive function, with both too little and too much activity hurting performance.

for T2, rendering its representation vulnerable to distractor interference (Chun \& Potter, 1995; Shapiro, Raymond, \& Arnell, 1994).

However, limited-capacity theories of the $\mathrm{AB}$ were challenged by results showing that participants have no trouble reporting three or more consecutive targets as long as there are no intervening distractors (Di Lollo, Kawahara, Ghorashi, \& Enns, 2005; see also Olivers, Van Der Stigchel, \& Hulleman, 2007; Kawahara, Enns, \& Di Lollo, 2006; Kawahara, Kumada, \& Di Lollo, 2006; Nieuwenstein, 2006; Nieuwenstein \& Potter, 2006). This observation is difficult to explain from a limited capacity perspective alone and spurred the development of novel accounts of the $\mathrm{AB}$, which assign greater importance to the first distractor stimulus after $\mathrm{T} 1$ and explain the $\mathrm{AB}$ in terms of dysfunctional gating of information into WM, rather than a capacity limitation of WM per se (Olivers \& Meeter, 2008; Di Lollo et al., 2005).

Findings from neuroimaging and electrophysiological studies confirm the critical role of WM in the AB. For example, activity in brain areas crucial for WM processes, such as lateral frontal and parietal cortex, reliably differentiates between trials in which T2 is seen (no-blink trials) versus missed (blink trials; Slagter, Johnstone, 
Beets, \& Davidson, 2010; Kranczioch, Debener, Schwarzbach, Goebel, \& Engel, 2005; Gross et al., 2004; Marois, Chun, \& Gore, 2000). Especially, left dorsolateral pFC (DLPFC) seems to be a critical node in the AB (Slagter et al., 2010; Hommel et al., 2006). Slagter et al. (2010), for example, found that, across participants, greater $\mathrm{T} 2$ detection-related activity in left DLPFC was associated with a smaller AB. This is notable because left DLPFC is deemed particularly important in situations that require maintenance of target information in WM in the face of distraction (Feredoes, Heinen, Weiskopf, Ruff, \& Driver, 2011; McNab \& Klingberg, 2008; Postle, 2006), as is the case in the AB task. Furthermore, this finding suggests that differences in left DLPFC activation might contribute to individual differences in $\mathrm{AB}$ magnitude. One consistent observation in the literature is that individuals differ substantially in the size of their $\mathrm{AB}$; some display a very big $\mathrm{AB}$ and almost always miss T2, whereas others virtually always detect T2 (i.e., display no AB; Martens, Munneke, Smid, \& Johnson, 2006), with most individuals falling somewhere between these two extremes (Dale, Dux, \& Arnell, 2013). Activity levels in left DLPFC may underlie these individual differences in $\mathrm{AB}$ magnitude and in target selection more generally.

In this study, we used transcranial direct current stimulation (tDCS) over left DLPFC to modulate the excitability of this brain region and examine if it is causally involved in the $\mathrm{AB}$. The reference electrode was placed over right anterior pFC (aPFC). In tDCS, a weak and constant electric current is delivered to the brain using two electrodes attached to the scalp. Current flows from the positive (anodal) to the negative (cathodal) electrode. The excitability of cortical areas below the anodal electrode is increased, whereas areas below the cathodal electrode decrease in excitability (Nitsche \& Paulus, 2000; Bindman, Lippold, \& Redfearn, 1962). An important difference between tDCS and TMS, another widely used stimulation technique, is that it does not directly induce neuronal action potentials. Rather, tDCS makes the endogenous generation of action potentials more or less likely by tonically depolarizing or hyperpolarizing the resting membrane potential (Nitsche et al., 2008). In addition to these immediate effects, long-term effects of up to $1.5 \mathrm{hr}$ after stimulation have been shown for stimulation durations of $9 \mathrm{~min}$ and longer in the motor domain (Nitsche \& Paulus, 2001). Recent work furthermore suggests that stimulation effects on performance are at least in part determined by the preexisting balance between cortical excitation and inhibition (Krause \& Cohen Kadosh, 2014; Krause, Márquez-Ruiz, \& Cohen Kadosh, 2013). The relation between the excitationinhibition balance within a brain area and its efficiency is argued to follow an inverted-U shape in which functioning is optimal when excitation and inhibition interact in a way that permits both flexibility (i.e., plasticity, new learning) and stability (i.e., resistance to distraction, maintenance of information).
Baseline excitation-inhibition balances might, however, vary across individuals within a given brain region and determine the effect of brain stimulation depending on whether it moves the balance toward or away from its optimum. For example, if a certain brain area is functioning optimally, anodal tDCS (atDCS) would worsen its efficiency because of overexcitation. Conversely, if the area functions suboptimally because of overinhibition, atDCS would improve its efficiency. Thus, atDCS may improve performance in individuals with suboptimal levels of cortical excitability, while impairing performance in individuals with optimal or supra-optimal cortical excitability by causing overexcitation.

Given that individual differences in $\mathrm{AB}$ magnitude have been linked to individual differences in left DLPFC activation (Slagter et al., 2010) and the fact that stimulation effects on performance may depend on baseline brain excitability levels, we hypothesized that atDCS over left DLPFC would modulate the $\mathrm{AB}$ as a function of individual baseline $\mathrm{AB}$ magnitude. Specifically, our main prediction was that individuals with a relatively large $A B$ to begin with would benefit most from anodal stimulation, whereas those with a small baseline $\mathrm{AB}$ would benefit less or even display a decrement in performance as a result of anodal stimulation. We did not expect an effect of cathodal tDCS (ctDCS) given previous work using a similar stimulation protocol showing no differential effect of cathodal versus sham stimulation of left DLPFC on WM performance (Fregni et al., 2005).

To control for polarity-unrelated effects of tDCS, we applied both atDCS and ctDCS over left DLPFC. If the mere fact of receiving tDCS would systematically influence performance on the $\mathrm{AB}$ task, for example, by distracting the subject and producing a more diffuse attentional set (Arend, Johnston, \& Shapiro, 2006; Olivers $\&$ Nieuwenhuis, 2006), then we would expect similar results for both stimulation conditions. If, however, only atDCS affects performance, we can exclude the possibility that such nonspecific effects contributed to our findings and safely conclude that $\mathrm{AB}$ performance was modulated by anodal electrical stimulation.

In addition to examining the effects of tDCS on T2 identification, we looked at how tDCS affects the ability to inhibit irrelevant distractors. Distractor inhibition appears to play an important role in the AB (Arnell \& Stubitz, 2010; Olivers \& Meeter, 2008; Di Lollo et al., 2005; Chun \& Potter, 1995), and evidence suggests that left DPLFC may be particularly involved in situations in which target information has to be maintained in WM in the face of distraction (Feredoes et al., 2011; McNab \& Klingberg, 2008; Postle, 2006). It is thus possible that any observed stimulation-induced modulations of the $\mathrm{AB}$ reflect a changed ability to inhibit distracting information. To explore the role of left DLPFC in distractor inhibition and enhance our understanding of how this area is involved in the $A B$, we used a version of the $A B$ task designed by Dux and Marois (2008) that permitted us to quantify not only participants' $\mathrm{AB}$ performance but also 


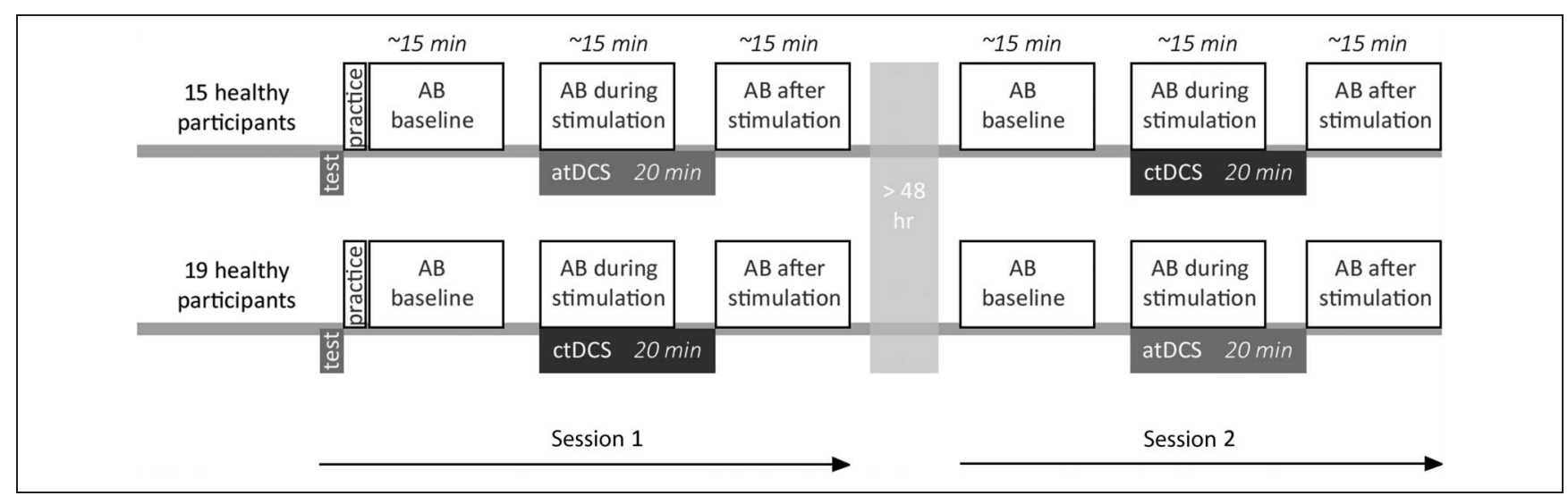

Figure 1. Experimental procedure. Participants completed two sessions that differed only in the polarity of the tDCS administered (atDCS or ctDCS). In each session, participants performed three blocks of 200 trials of the AB task: one before tDCS (baseline), one during the 20-min stimulation period (during tDCS), and one after stimulation ended (after tDCS). Sessions were at least $48 \mathrm{hr}$ apart.

their ability to inhibit distractor stimuli. Specifically, distractor inhibition was inferred by examining the influence of a post-T1 distractor that primed $\mathrm{T} 2$ on subsequent T2 identification. More effective distractor inhibition should lead to a prime-related reduction in T2 identification because strong suppression of the prime stimulus should render reactivation of this representation by $\mathrm{T} 2$ more difficult. Conversely, less effective distractor inhibition should lead to a prime-related enhancement in T2 identification because the representation of $\mathrm{T} 2$ benefits from residual activation of its identity by the prime. If anodal stimulation over left DLPFC improves or disrupts distractor inhibition, one would thus expect to see a smaller or larger priming effect, respectively. Furthermore, if distractor inhibition is an important determinant of the $\mathrm{AB}$, one may predict that anodal-stimulationinduced changes in distractor inhibition lead to changes in $\mathrm{AB}$ performance.

Thus, the current study examined the effects of atDCS versus ctDCS over left DLPFC on the $A B$ and on distractor inhibition. Our main hypothesis was that atDCS, but not ctDCS, over left DLPFC would modulate the AB as a function of individual baseline $\mathrm{AB}$ magnitude and by changing participants' ability to inhibit distracting information.

\section{METHODS}

\section{Participants}

Thirty-eight participants ( 22 women, mean age $=22.4$ years, $S D=2.8$ years), of which four were later excluded (see Results), took part in the study. All had normal or corrected-to-normal sight, had no history of neurological or psychiatric disorders, and were not color blind. The study was approved by the local ethics committee. Participants gave written informed consent and were compensated with course credit or money (10 Euros per hour). Four participants (two women) were excluded because of poor T1 identification (see below).

\section{Experimental Procedure}

Participants performed an $\mathrm{AB}$ task in two sessions, one in which they received anodal stimulation and one in which they received cathodal stimulation (see Figure 1 for an overview of the experimental design). These two sessions were separated by at least $48 \mathrm{hr}$ to ensure that the effects of the previous session had washed out (Nitsche \& Paulus, 2001). Session order was counterbalanced across participants, such that 15 participants received atDCS in the first session and ctDCS in the second session and 19 participants received ctDCS in the first session and atDCS in the second session. At the beginning of the first session, participants received 1-mA atDCS to left DLPFC for $15 \mathrm{sec}$, ramped up and down in $30 \mathrm{sec}$, so that they could briefly experience the sensation before beginning the experiment and decide whether they wanted to continue. All participants decided to continue with the experiment. During each session, participants first briefly practiced the $\mathrm{AB}$ task and then performed the $\mathrm{AB}$ task before stimulation (baseline), during stimulation, and again after stimulation. After each session, 33 of 34 participants completed questionnaires on possible physical side effects of the stimulation. In addition, before and after each session, of these 33 participants, 31 completed the short form of the Activation-Deactivation Adjective Check List (AD ACL) questionnaire designed to assess various arousal states and mood at the present moment (Thayer, 1978). The test-retest reliability of its four subscales is high: energy $=.92$, tension $=.89$, calmness $=$ .89 , and tiredness $=.90$.

\section{tDCS}

tDCS was delivered with a battery-driven, constant current stimulator (Neuroconn, Ilmenau, Germany) with a maximum output of $10 \mathrm{~mA}$ and administered by two $35-\mathrm{cm}^{2}(5 \times 7)$ galvanized rubber electrodes inside saline-soaked sponges that were placed on the scalp 
with rubber bands. The electrode of interest was placed over left DLPFC at the F3 position according to the 1020 system for EEG electrode placement (Committee on Methods of Clinical Examination in Electroencephalography, 1957; DaSilva, Volz, Bikson, \& Fregni, 2011). The reference electrode was placed on the contralateral supraorbital area of the face, over right aPFC. This electrode setup is similar to previous studies, which reported effects of atDCS on WM performance (Keeser et al., 2011; Teo, Hoy, Daskalakis, \& Fitzgerald, 2011; Fregni et al., 2005). In the anodal stimulation condition, participants received 1-mA stimulation over left DLPFC for $20 \mathrm{~min}$. In the cathodal stimulation condition, participants received $-1-\mathrm{mA}$ stimulation over left DLPFC for $20 \mathrm{~min}$. In both conditions, the current was ramped up in $20 \mathrm{sec}$ and ramped down in $60 \mathrm{sec}$.

\section{Task and Design}

The task was identical to the task used by Slagter and Georgopoulou (2013) and modeled after the task designed by Dux and Marois (2008; see Figure 2). Each trial consisted of a rapid serial visual presentation (RSVP) of 17 uppercase letters excluding I, L, O, Q, U, and V (font type: Courier New, font size: 40). T1 was red, T2 was green, the distractors were white, and the background was gray. Participants were instructed to detect both targets. T1 appeared at Serial position 5, and T2 followed T1 after one, three, or nine distractors, that is, at Lags 2, 4, and 10, respectively. Each trial started with a fixation

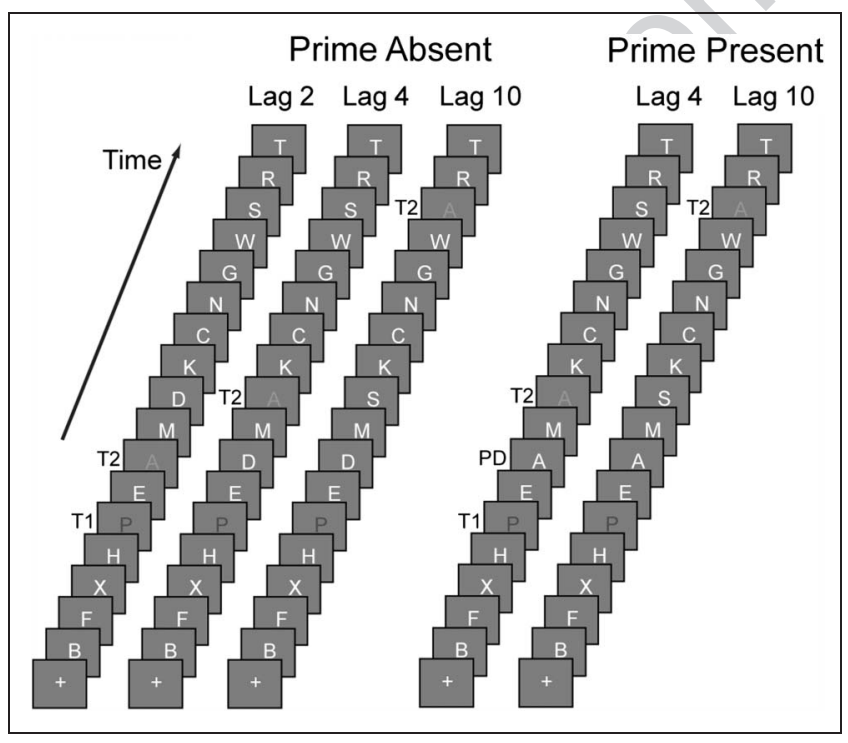

Figure 2. AB task. Participants viewed RSVP streams of letters. T1 was red (here displayed in dark gray), T2 was green (here displayed in light gray), and the distractors were white. T2 could appear at Lag 2, 4, or 10. In the prime-present trials, a priming distractor with the same identity as T2 appeared at Lag 2. All stimuli had different identities in the prime-absent trials. Participants reported the identities of T1 and $\mathrm{T} 2$ at the end of each stream. square presented for $480 \mathrm{msec}$, followed by the 17 letters, each presented for $92 \mathrm{msec}$. In prime-absent trials, all stimuli were different, whereas in the prime-present trials, the second distractor after T1 (i.e., at Lag 2) had the same identity as $\mathrm{T} 2$ (priming distractor). This distractor was presented at the lag where the $A B$ is typically maximal, making it unlikely that it was consciously perceived. Necessarily, priming distractors could only occur in trials in which $\mathrm{T} 2$ was presented at Lag 4 or Lag 10. Thus, there were five trial types: prime-absent Lag 2, prime-absent Lag 4, prime-absent Lag 10, prime-present Lag 4, and prime-present Lag 10. At the end of each RSVP, participants were prompted to input the target identities using a keyboard. The task was programmed using Presentation software. Participants were seated approximately $90 \mathrm{~cm}$ from a computer screen in a comfortable chair. The 23-in. LCD highperformance gaming monitor was driven by a standard personal computer running the Microsoft operating system XP and refreshed at $120 \mathrm{~Hz}$ with a resolution of $1920 \times 1080$ pixels in 16-bit color.

In each session, participants performed three blocks of 200 trials of the $\mathrm{AB}$ task (approximately $15 \mathrm{~min}$ per block): a block before tDCS stimulation (baseline), a block during the 20-min stimulation period (during tDCS), and a block after stimulation ended (after tDCS; see Figure 1). Trial types were equally probable and randomly intermixed within a block. Participants thus performed 40 trials in each block of each trial type. Before each baseline block, participants performed 20 practice trials. The baseline blocks were included in each session to exclude possible differences between sessions in situational factors such as amount of sleep and mood from affecting our results, as both arousal and mood have been shown to affect the magnitude of the $\mathrm{AB}$ (Jefferies, Smilek, Eich, \& Enns, 2008; Olivers \& Nieuwenhuis, 2006).

\section{Questionnaires}

The questionnaire on the possible side effects of tDCS consisted of eight items describing physical sensations for which participants were asked to indicate to what extent each had been present during stimulation. The eight physical sensations were itching, prickling, burning, pain, headache, fatigue, dizziness, and nausea. Response options were "not," "a little," "somewhat," "strongly," or "very strongly."

The Short Form AD ACL consists of 20 items on four subscales for which participants had to indicate whether they felt like this "definitely not," "not really," "a little," or "definitely" (Thayer, 1978). The feelings probed were active, energetic, vigorous, lively, and full of pep (subscale energy); sleepy, tired, drowsy, wide awake, and wakeful (subscale tired); jittery, intense, fearful, clutched up, and tense (subscale tension); and placid, calm, at rest, still, and quiet (subscale calmness). 


\section{Analyses}

\section{Individual Differences}

To examine whether the effects of tDCS depended on individual differences in baseline $\mathrm{AB}$ performance and/ or distractor inhibition ability, we first calculated two parameters: $\mathrm{AB}$ magnitude and $\mathrm{T} 2$ priming. This was done for each participant separately for each block (before, during, and after tDCS) and stimulation session (anodal and cathodal). AB magnitude was calculated as T2 accuracy given that T1 was correct (T2|T1) in Lag 10 versus Lag 2 prime-absent trials. T2|T1 accuracy was assessed in prime-absent trials to get a measure of $\mathrm{AB}$ magnitude that was independent of the prime (Slagter \& Georgopoulou, 2013; Dux \& Marois, 2008). T2 priming, from which the amount of distractor inhibition was inferred, was quantified as T2|T1 in Lag 4 prime-present trials versus Lag 4 prime-absent trials. T2 priming was only assessed at Lag 4, and not at Lag 10, because of the short duration of RSVP priming (Maki, Frigen, \& Paulson, 1997). To assess if the effects of tDCS depended on individual differences in baseline $\mathrm{AB}$ performance or baseline distractor inhibition ability, we then calculated the change from baseline, separately for $\mathrm{AB}$ magnitude and $\mathrm{T} 2$ priming and the blocks of during and after atDCS and ctDCS, by subtracting the respective baseline scores from the scores in each of these four blocks. Partial Pearson correlations between these change measures (e.g., $\mathrm{AB}$ magnitude during atDCS and before atDCS) and their corresponding baselines (i.e., $\mathrm{AB}$ magnitude before atDCS) were then used to estimate if effects of tDCS on performance during and after stimulation depended on initial performance. In these analyses, we controlled for session order and $\mathrm{T} 1$ performance to exclude the possibility that observed effects could simply be explained by practice effects or an overall change in target identification, respectively. Alpha (.05) was divided by four to account for the number of correlation tests used to assess the relationships between baseline performance and stimulation-related change in performance for both $\mathrm{AB}$ magnitude and T2 priming. Thus, an alpha of .0125 was applied.

Finally, we applied a test for equality of variances to those variables of interest (Myrtek \& Foerster, 1986) that were significantly correlated, to exclude regression to the mean as an alternative explanation.

\section{Group Level}

$A B$ task. We next examined if tDCS also affected $A B$ performance and/or distractor inhibition at the group level. For each participant, lag (2, 4, and 10), block (baseline, during tDCS, and after tDCS), and stimulation condition (atDCS and ctDCS) separately, the percentage of trials in which T1 was accurately identified (regardless of T2 performance) and the percentage of trials in which both targets were correctly identified (T2|T1 accuracy) were calculated. To investigate the effect of tDCS on the $\mathrm{AB}$, a repeated-measures ANOVA with $\mathrm{T} 2 \mid \mathrm{T} 1$ accuracy in prime-absent trials as the dependent variable was conducted with lag (2, 4, and 10), block (baseline, during, and after), and stimulation (atDCS and ctDCS) as within-subject factors and session order (atDCS first and ctDCS first) as a between-subject factor. A similar analysis was run with T1 accuracy as the dependent variable to assess effects of stimulation on T1 performance. The effect of tDCS on distractor inhibition was tested with a repeated-measures ANOVA with prime (absent and present), lag ( 4 and 10), block (baseline, during, and after), and stimulation (atDCS and ctDCS) as within-subject factors and session order (atDCS first and ctDCS first) as a between-subject factor.

Questionnaires. To examine whether there were systematical differences in physical sensations between atDCS and ctDCS, paired-sample $t$ tests were conducted for each of the eight items on the tDCS side-effects questionnaire. To determine whether there was a difference in the effects of atDCS versus ctDCS on arousal states, scores on each of the four subscales were calculated before and after stimulation for each stimulation session separately and subsequently subtracted from each other to obtain a measure of the effect of electrical stimulation. For each subscale separately, a paired-sample $t$ test was then conducted comparing the resulting difference scores between the atDCS and ctDCS conditions. A Bonferroni correction was applied to account for multiple comparisons for both questionnaires separately resulting in an alpha of $.05 / 8=.0063$ for the tDCS side-effects questionnaire and an alpha of $.05 / 4=.0125$ for the Short Form $\mathrm{AD}$ ACL questionnaire.

\section{RESULTS}

Four participants (two women) were excluded because of poor T1 identification ( $>2 S D$ s below the mean). In the remaining 34 participants, average T1 accuracy over both baseline measurements was $86 \%$.

\section{Individual Differences}

Replicating previous reports, large individual differences in $\mathrm{AB}$ magnitude (Dale et al., 2013) and distractor inhibition (Slagter \& Georgopoulou, 2013; Dux \& Marois, 2008) were observed.

\section{Individual Differences in Baseline AB Magnitude Predicted Effects of Anodal, but Not Cathodal, tDCS on AB Performance}

Our main prediction was that anodal stimulation would decrease the $\mathrm{AB}$ in individuals who have a relatively large $A B$ to begin with, whereas it would increase the $A B$ in 

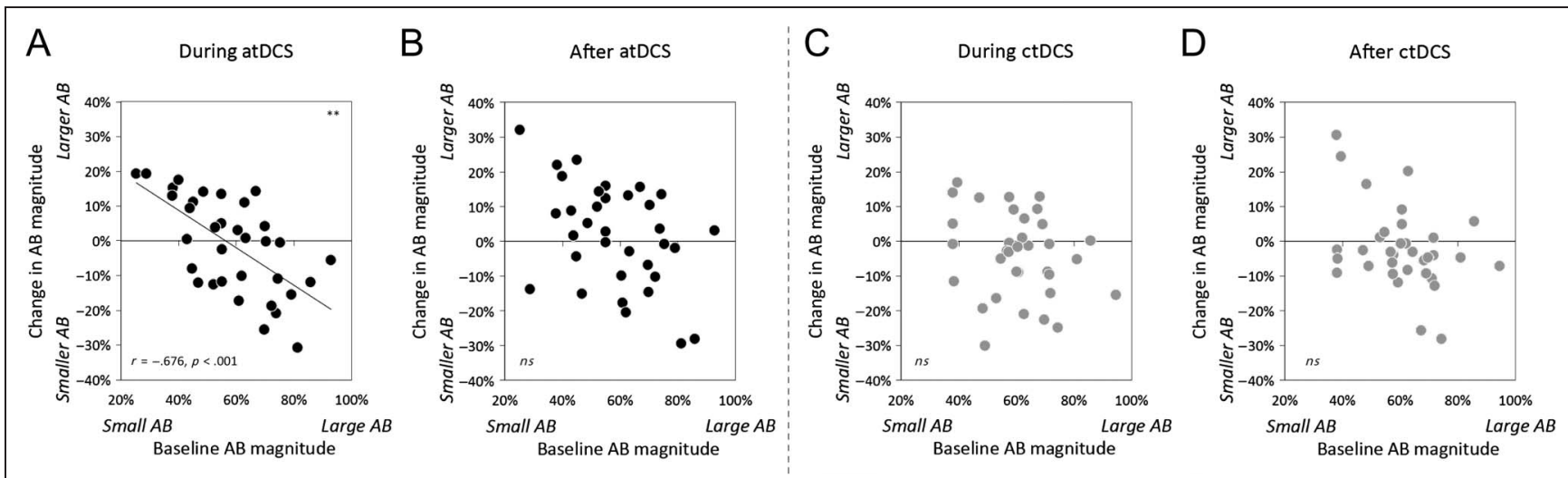

Figure 3. Baseline $A B$ magnitude selectively predicted the effects of atDCS on the AB across participants during atDCS (A), but not after atDCS (B). This effect could not simply be explained by regression to the mean. No significant relationship between baseline AB magnitude and effects of cathodal stimulation on the $\mathrm{AB}$ was observed during (C) or after (D) cathodal stimulation. $* * p<.001 . n s=$ nonsignificant.

individuals with a relatively small baseline $\mathrm{AB}$. To test this prediction, we correlated individual baseline $\mathrm{AB}$ magnitude with the change in $\mathrm{AB}$ magnitude during (or after) stimulation versus baseline, while controlling for session order and possible stimulation-related changes in T1 accuracy at Lag 2, separately for atDCS and ctDCS. As predicted, we found a significant inverse relation between baseline $\mathrm{AB}$ magnitude and change in $\mathrm{AB}$ magnitude during atDCS $(r(30)=-.676, p<.001$; see Figure $3 \mathrm{~A})$. Importantly, this relationship was not present in the cathodal stimulation condition $(r(30)=-.230$, $p=.22$; see Figure $3 \mathrm{C}$ ). Confirming the specificity of the relationship between the effect of anodal (but not cathodal) stimulation and individual baseline $\mathrm{AB}$ magnitude, a Pearson-Filon test (Raghunathan, Rosenthal, $\&$ Rubin, 1996) showed that these two correlations were indeed significantly different from each other $(z=$ $-2.00, p<.05)$. Thus, individuals with a relatively large $\mathrm{AB}$ to begin with appeared to benefit most from atDCS over left DLPFC, whereas those with a relatively small baseline $\mathrm{AB}$ tended to benefit less or even exhibit a larger $\mathrm{AB}$. This relationship was not observed during ctDCS.

Of further importance, the observed relationship between baseline $\mathrm{AB}$ magnitude and the effect of atDCS on the $\mathrm{AB}$ could not be explained by regression to the mean. Measurement error is always a concern in analyses examining the relationship between a baseline measure and the change in this measure over time. It is well known that, if the variable has an extreme value when first measured, it will tend to be closer to the mean when measured on a second occasion and, if it has an extreme value during the second measurement, it will tend to have been closer to the mean at first. This problem is known as regression to the mean. Moreover, even when no relationship exists between the baseline $(x)$ and the change $(y-x)$, the fact that $x$ is present in both terms leads to an expected spurious correlation of -.7 between $x$ and $y-x$ (Tu \& Gilthorpe, 2007). To address these issues and rule out regression to the mean as an alternative explanation for the observed relationship between baseline $\mathrm{AB}$ magnitude and the change in $\mathrm{AB}$ magnitude induced by atDCS, we applied a procedure designed to test the equality of variances between the two conditions (Tu \& Gilthorpe, 2007; Jin, 1992; Myrtek \& Foerster, 1986). If participants with a large $A B$ indeed benefit from atDCS whereas participants with a small $\mathrm{AB}$ suffer, the variance of $\mathrm{AB}$ scores during baseline testing should be significantly higher than that during atDCS. In contrast, if there is no effect of stimulation, then variance should remain the same from one measurement to the next. Indeed, we found that variance of baseline $A B$ scores (0.026) was significantly higher than variance of $\mathrm{AB}$ scores during atDCS $(0.017 ; t(32)=3.88, p<.001)$. Thus, critically, we can rule out regression to the mean as an explanation for our observation that individuals who had a relatively large baseline $\mathrm{AB}$ benefited from atDCS over left DLPFC in combination with ctDCS over right aPFC, whereas those with a small baseline $\mathrm{AB}$ benefited less or even exhibited a decrement in performance. One alternative explanation of the observed lower variance during anodal stimulation compared with the preceding baseline block is stabilization of performance with practice. If so, one would also expect a reduction in variance of $\mathrm{AB}$ scores during ctDCS compared with baseline. Ruling out this alternative possibility, variance in $\mathrm{AB}$ size was higher during ctDCS (0.025) compared with baseline $(0.019 ; t(32)=2.77, p<.01)$.

Of further note, baseline $\mathrm{AB}$ magnitude only predicted the change in $\mathrm{AB}$ magnitude during, but not after, atDCS. Although a modest correlation was observed between baseline $\mathrm{AB}$ and the postanodal stimulation change in $\mathrm{AB}$ magnitude $(r(30)=-.427, p=.019$; Figure $3 \mathrm{~B})$, it did not survive correction for multiple comparisons. Moreover, the test for equality of variances was not significant $(t(32)=0.23, p=.822)$, rendering it unclear if it reflects a true relationship or regression to the mean. In the cathodal condition, the correlation between baseline and poststimulation effect was also not significant after correction for multiple comparisons $(r(30)=-.381$, $p=.038$; Figure 3D). 
To summarize, individual differences' analyses revealed that, as predicted, atDCS over left DLPFC with ctDCS over right aPFC decreased the $\mathrm{AB}$ in individuals with a large baseline $A B$ but increased the $A B$ in individuals with a small baseline $\mathrm{AB}$. This effect was only observed during (but not after) stimulation, was not found for ctDCS over left DLPFC with atDCS over right aPFC, and could not be explained by regression to the mean. These findings support the idea that pFC plays a critical role in the AB. They are also in line with the notion that there is an optimum level of cortical activity for cognitive function, with both too little and too much activity hurting performance. On the basis of this line of thought, one would also predict that the effects of anodal and cathodal stimulations on $\mathrm{AB}$ magnitude for a given individual would go in opposite directions. We explored this in a post hoc analysis in which we correlated the atDCS-induced change in $\mathrm{AB}$ magnitude with the ctDCSinduced change in $\mathrm{AB}$ magnitude across participants, while controlling for session order. This analysis revealed a significant negative relationship $(r(31)=-.445, p<$ .01 ; see Figure 4 ), indicating that individuals who benefited from atDCS generally worsened because of ctDCS, whereas individuals who worsened because of atDCS tended to perform better because of ctDCS.

Individual Differences in Baseline Distractor Inbibition Are Not Predictive of the Effect of Stimulation on Distractor Inbibition

We next investigated whether effects of stimulation on distractor inhibition depended on an individual's baseline

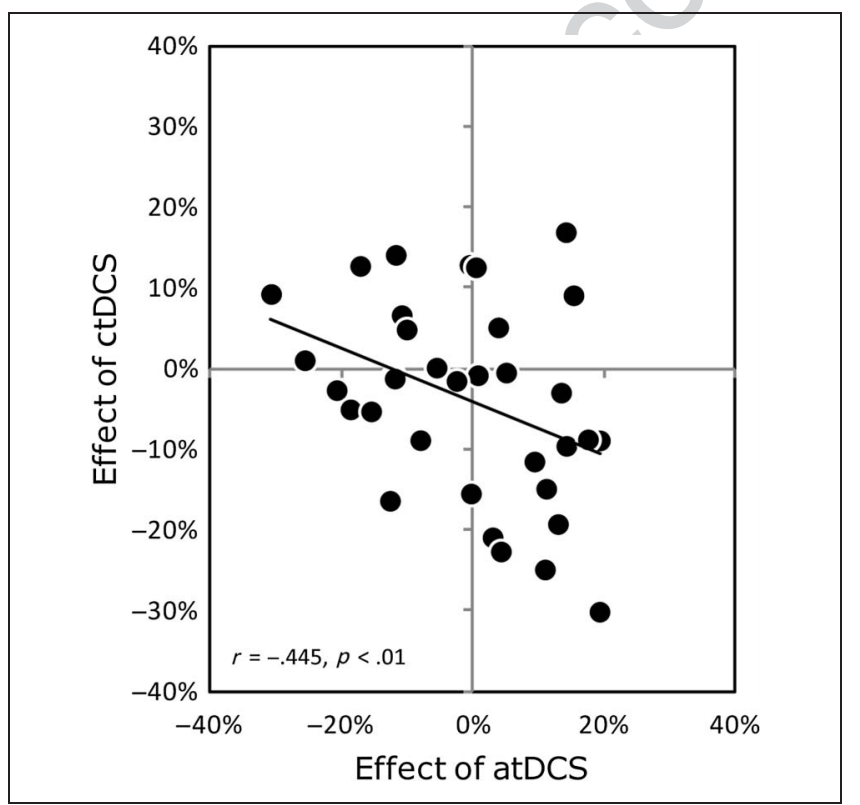

Figure 4. The effects of atDCS and ctDCS opposed each other. Participants who improved with atDCS tended to get worse with ctDCS and vice versa. ability to inhibit distractors. To this end, we tested the relation between baseline $\mathrm{T} 2$ priming and the changes in T2 priming during and after atDCS and ctDCS, controlling for session order and for change in T1 accuracy at Lag 4 averaged over prime-present and prime-absent trials. Significant negative correlations were obtained for all tests conducted; baseline distractor priming change in distractor priming during atDCS $(r(30)=$ $-.723, p<.001)$, during ctDCS $(r(30)=-.578, p<$ $.005)$, after atDCS $(r(30)=-.543, p<.005)$, and after $\operatorname{ctDCS}(r(30)=-.789, p<.001)$. However, none of these correlations survived the test for equality of variances $(t(32)=0.84, p=.406 ; t(32)=-0.50, p=.617 ; t(32)=$ $0.12, p=.908 ; t(32)=0.80, p=.430$, respectively), indicating that we cannot exclude the possibility that these correlations can simply be explained by regression to the mean.

Furthermore, we post hoc examined the reliability of our measure of distractor inhibition. To this end, we correlated the baseline priming effect measured in the two separate sessions across participants using a Pearson correlation test. Surprisingly, the baseline priming effect was not correlated between sessions $(r(31)=-.089$, $p=.64$; controlling for session order), suggesting that this measure does not reflect a stable trait and calling for caution when interpreting the results of analyses including this measure. Given the unreliability of the priming measure, the fact that observed stimulation-induced changes in priming may simply reflect regression to the mean, and the fact that priming did not predict $\mathrm{AB}$ magnitude in a previous study (Slagter \& Georgopoulou, 2013), we did not further examine if stimulation-induced changes in $\mathrm{AB}$ magnitude could be explained by stimulationinduced changes in distractor inhibition ability, as indexed by this priming measure. Importantly, baseline $\mathrm{AB}$ magnitude was reliably correlated between sessions $(r(31)=$ $.583, p<.001$; controlling for session order), replicating previous reports that $\mathrm{AB}$ performance is stable over time (Dale et al., 2013).

\section{Group Results}

\section{Effects of tDCS on the $A B$}

Next, we investigated effects of tDCS on the magnitude of the $\mathrm{AB}$ at the group level. As can be seen in Figure 5, on average, participants displayed a clear $\mathrm{AB}$, both before, during, and after atDCS and ctDCS (significant main effect of lag: $F(2,64)=311.80, p<.001)$, but at the group level, tDCS did not modulate the size of the $A B$ : No differential effect of atDCS versus ctDCS on the $\mathrm{AB}$ was observed, as indexed by a nonsignificant interaction between stimulation, lag, and block $(F(4,128)=$ $0.829, p=.509$ ).

Session order did not influence the effect of stimulation on the $\mathrm{AB}$ but did affect $\mathrm{T} 2$ performance in general (regardless of lag), as indicated by a significant interaction between 


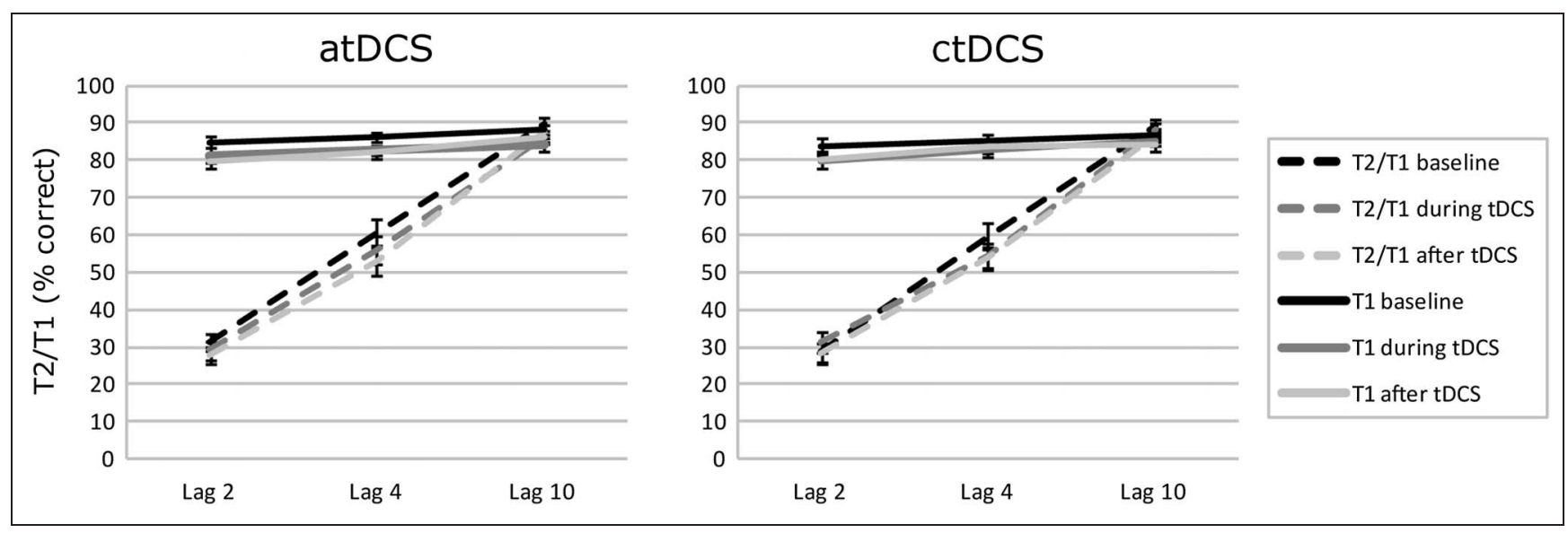

Figure 5. Group effects of atDCS and ctDCS on T1 performance (solid lines) and T2/T1 performance (dotted lines) in prime-absent trials. At the group level, anodal nor cathodal stimulation affected T1 accuracy or the AB.

session order and stimulation $(F(1,32)=17.05, p<.001)$. Specifically, participants who received atDCS in the first session, on average, showed a greater improvement in T2|T1 accuracy (regardless of lag) from the first session to the second session than those who received ctDCS in the first session. Moreover, a main effect of block $(F(2,64)=9.14, p<.001)$ was found reflecting a decrease in $\mathrm{T} 2 \mid \mathrm{T} 1$ performance over time. Yet, importantly, this effect was not modulated by the type of stimulation applied (nonsignificant interaction between block and stimulation: $F(2,64)=1.50, p=.231)$.

With regard to T1 accuracy, as can be seen in Figure 5, atDCS also did not affect T1 performance at the group level, as reflected in nonsignificant interaction effects between block and stimulation $(F(2,64)=0.084, p=$ .919) and stimulation, lag, and block $(F(4,128)=1.17$, $p=.329)$. Again, a main effect of block $(F(2,64)=$ $11.90, p<.001)$ was found, reflecting decreasing T1 accuracy over time, which was also observed regardless of the type of stimulation applied (reflected in the nonsignificant interaction between block and stimulation). Thus, $\mathrm{T} 1$ and $\mathrm{T} 2$ performance both displayed a generally decline over time, perhaps reflecting a time-on-task effect and/or polarity-unrelated effects of tDCS, such as fatigue or headache (Brunoni et al., 2011; Poreisz, Boros, Antal, \& Paulus, 2007). T1 performance was also affected by lag (significant main effect of lag: $F(2,64)=20.56, p<$ .001 ), but this effect was not modulated by the polarity of stimulation as reflected by the nonsignificant interaction between stimulation, lag, and block $(F(4,128)=$ $1.17, p=.329$ ). To summarize these findings, at the group level, atDCS nor ctDCS over left DLPFC affected the performance for $\mathrm{T} 1$ or $\mathrm{T} 2$.

\section{tDCS Side Effects and Effects on Arousal}

To rule out the possibility that participants were differentially affected by the physical sensations brought on by the two stimulation conditions, we compared self- reported intensities of eight physical sensations during atDCS and ctDCS. Paired-sample $t$ tests revealed that participants did not experience significantly different physical side effects during atDCS compared with ctDCS: itching, $t(32)=-0.571, p=.572$; prickling, $t(32)=$ 2.390, $p=.023$; burning, $t(32)=0.865, p=.394$; pain, $t(32)=1.75, p=.090$; headache, $t(32)=0.000, p=1$; fatigue, $t(32)=-0.130, p=.897$; dizziness, $t(32)=0.000$, $p=1$; nausea, $t(32)=-1.000, p=.325$.

Next, we examined if there was a different effect on arousal levels of atDCS compared with ctDCS. No significant differences were found on any of the subscales: energy, $t(30)=0.345, p=.733$; tired, $t(30)=-0.635, p=$ .530; tension, $t(30)=-0.879, p=.387$; calmness, $t(30)=$ $-0.894, p=.378$.

\section{GENERAL DISCUSSION}

The current study examined the effects of tDCS on the $\mathrm{AB}$ and on distractor inhibition. Our main hypothesis was that atDCS, but not ctDCS, over left DLPFC would modulate the $\mathrm{AB}$ as a function of individual baseline $\mathrm{AB}$ magnitude and by modifying participants' ability to filter out distracting information. Indeed, individual differences' analyses revealed that atDCS decreased the $A B$ in individuals with a large baseline $A B$ but increased the $\mathrm{AB}$ in individuals with a small baseline $\mathrm{AB}$. This effect was only observed during (but not after) anodal stimulation, was not found for ctDCS, and importantly, could not be explained by regression to the mean. No effects of tDCS were observed at the group level, emphasizing the importance of taking individual differences in baseline performance into account, as others have recently argued (Benwell, Learmonth, Miniussi, Harvey, \& Thut, 2015; Li, Uehara, \& Hanakawa, 2015). tDCS did not affect distractor inhibition, but post hoc analyses revealed low test-retest reliability of our priming measure, rendering it difficult to interpret this result. Below, these findings are discussed in more detail. 
Our observation that atDCS generally reduced the $\mathrm{AB}$ in individuals with a large baseline $\mathrm{AB}$ but increased the $\mathrm{AB}$ in individuals with a small baseline $\mathrm{AB}$, whereas ctDCS had no significant effect on the $\mathrm{AB}$, adds to a growing body of work indicating that the idea that atDCS enhances cortical excitation and thereby performance (Nitsche \& Paulus, 2001) and ctDCS reduces cortical excitability and thereby may impair performance (Nitsche et al., 2003) is overly simplistic. First, similar to the current study, Fregni et al. (2005) only observed a modulation of verbal WM performance after atDCS, but not after ctDCS, over left DLPFC (Fregni et al., 2005). Thus, ctDCS may not always modulate performance. Moreover, as in this study, a growing number of studies report that the effects of stimulation on behavior can vary considerably from individual to individual (Benwell et al., 2015; Krause \& Cohen Kadosh, 2014; López-Alonso, Cheeran, RíoRodríguez, \& Fernández-Del-Olmo, 2014; for a review, see Li et al., 2015). Thus, the effects of tDCS on behavior are complex and are difficult to predict without taking into account preexisting individual differences. In fact, as mentioned above, the effects of tDCS were not apparent at the group level. These results are a clear example of how effects of tDCS can be masked by individual differences in baseline performance.

This raises the important question of which factors determine how tDCS affects performance in a given individual. As noted in the Introduction, it has recently been suggested that interindividual variability in stimulation effects is at least in part related to interindividual variability in the balance between cortical excitation and inhibition (Krause et al., 2013) and that this balance modulates the effect of tDCS in a nonlinear fashion. Specifically, the relation between the excitation-inhibition balance within a brain area on the one hand, and its efficiency on the other, is suggested to follow an inverted-U shape in which optimal performance is achieved when excitation and inhibition interact in a way that permits both flexibility and stability. Thus, atDCS may have improved performance in individuals with suboptimal prefrontal excitability but impaired performance in individuals with optimal or supra-optimal prefrontal excitability because of overexcitation. Notably, although no significant relationship between baseline $\mathrm{AB}$ magnitude and the effect of ctDCS was observed, the negative relationship between the effect of atDCS and the effect of ctDCS was highly significant; participants who tended to benefit from atDCS generally worsened during ctDCS and vice versa. This finding provides indirect support for the existence of an inverted-U-shaped relationship between cortical excitation-inhibition balance and performance, which critically determines the effects of tDCS. Future studies that combine tDCS with neuroimaging techniques are necessary to investigate this idea more directly.

One of the factors influencing an individual's baseline excitation-inhibition balance of DLPFC is the neurotrans- mitter dopamine (Nitsche, Monte-Silva, Kuo, \& Paulus, 2010; Kuo, Paulus, \& Nitsche, 2008; Nitsche et al., 2006). Genetic variation is an important source of individual differences in baseline prefrontal dopamine levels (Dickinson \& Elvevåg, 2009) and activity patterns in pFC (Bertolino et al., 2006). Illustrating how this variation can modulate the effect of tDCS, Plewnia et al. (2013) showed that, in carriers of the COMT Met/Met allele, who tend to exhibit higher levels of prefrontal dopamine, atDCS was associated with a deterioration of cognitive performance. Nieratschker, Kiefer, Giel, Krüger, and Plewnia (2015) demonstrated that ctDCS, on the other hand, had a negative effect on cognitive performance in carriers of the COMT Val/Val allele, which is associated with lower levels of prefrontal dopamine. Moreover, dopamine has been shown to modulate cortical excitably in a nonlinear fashion and thereby influence effects of noninvasive brain stimulation (Nitsche et al., 2010). These studies are in line with the idea of an inverted-U-shaped relationship between the level of dopamine and cortical excitability on the one hand and cognitive performance on the other.

A largely separate literature on dopamine and cognition also supports the existence of a U-shaped relationship between prefrontal dopaminergic signaling and cognitive performance (Cools \& D'Esposito, 2011; Arnsten, 1998; Zahrt, Taylor, Mathew, \& Arnsten, 1997; Williams \& Goldman-Rakic, 1995). Specifically, this work shows that prefrontal dopamine levels are critical for cognitive stability or maintenance of information in WM and resistance to distraction and that both too low and too high levels of prefrontal dopamine can impair cognitive performance, albeit for different reasons. Whereas suboptimal prefrontal dopamine levels lead to a reduced ability to maintain information in WM for a brief period and greater distractibility (i.e., impaired cognitive stability), very high prefrontal dopamine levels can also impair performance by reducing one's ability to flexibly shift attention and dynamically update the contents of WM whenever novel relevant information occurs (i.e., impaired cognitive flexibility). Both a reduced ability to maintain target information online in face of distraction (because of overexcitation) and a reduced ability to flexibly update WM (because of overinhibition) could result in a larger $\mathrm{AB}$. Although we did not directly measure prefrontal excitability or dopamine levels, our observations that the effect of atDCS is dependent on baseline $\mathrm{AB}$ magnitude and that participants who tended to benefit from atDCS generally worsened during ctDCS and vice versa are in line with the existence of a U-shaped relationship between cognitive performance and prefrontal dopamine levels and excitability (Krause \& Cohen Kadosh, 2014; Cools \& D'Esposito, 2011). Other factors that might also have contributed to the observed individual differences in stimulation effects include individual differences in underlying brain morphology, scalp thickness, neurotransmitter balances, circadian rhythm, and hormonal levels (Kim et al., 2014; Krause \& Cohen Kadosh, 2014). Future studies that combine tDCS 
with measures of brain activity are necessary to further examine the complex relationship between baseline activity and effects of stimulation on performance (see also Horvath, Forte, \& Carter, 2014).

Our findings may corroborate previous studies showing that left DLPFC plays a critical role in the AB (Slagter et al., 2010; Kranczioch et al., 2005; Gross et al., 2004; Marois et al., 2000) and extend this work by providing causal evidence for a role of this region in the AB. However, one limiting factor of the current study is the inherent difficulty in separating the effects of the anodal and cathodal electrodes. In our case, the reference electrode was placed over right aPFC, and it is hence possible that the stimulation also affected activity in this region, in addition to left DLPFC. Yet, right aPFC has not, to our knowledge, been implicated in the $\mathrm{AB}$ in neuroimaging studies of the AB (Slagter et al., 2010; Kranczioch et al., 2005; Marois et al., 2000). In addition, previous research by Fregni et al. (2005) did not find significant effects of atDCS over right aPFC with the cathodal electrode over M1 nor of atDCS over right aPFC with the cathodal electrode over left DLPFC on WM performance compared with sham tDCS. That is, only atDCS over left DLPFC modulated WM performance relative to sham tDCS. Therefore, it is unlikely that stimulation of right aPFC contributed to our results. Nevertheless, based on the current data alone, this possibility cannot fully be excluded.

On a related note, studies modeling current flow show that the electric field generated by tDCS is highly nonspecific, involving widespread effects that differ over participants and are difficult to predict without explicit modeling based on anatomical data (e.g., Bikson \& Datta, 2012; Datta, Truong, Minhas, Parra, \& Bikson, 2012). Although electrode placement at the F3 site made it likely that left DLPFC was targeted (Sadleir, Vannorsdall, Schretlen, \& Gordon, 2010), we cannot exclude the possibility that, in some individuals, nearby structures were actually also and perhaps even more strongly affected than left DLPFC. Because the current had to make its way to the electrode placed over right aPFC, structures in its path must also have been affected, at least to some extent. Moreover, because there exist large differences in anatomy between individuals, the exact path the current took, and thereby the structures affected, likely differed between participants. This means that care must be taken to not draw too strong conclusions about the involvement of left DLPFC in the $\mathrm{AB}$ based on our results alone. However, our results do strongly suggest a critical role for $\mathrm{pFC}$ in the $\mathrm{AB}$, and left DLPFC is likely to be an especially important structure. This interpretation is in line with findings from fMRI and MEG studies of the $\mathrm{AB}$, which assign a particularly important role to left lateral pFC in the AB (e.g., Slagter et al., 2010; Hommel et al., 2006; Gross et al., 2004).

Ideally, for a study investigating the effects of an intervention such as tDCS, a placebo or sham condition is employed. In this case, however, we chose to compare
atDCS over left DLPFC with ctDCS over left DLPFC. It is possible that ctDCS and atDCS share nonspecific, polarity independent effects that are not shared by sham stimulation, in which case, ctDCS is a more suitable control than sham stimulation. Even so, a sham condition would have allowed us to assign our effects to anodal (and not cathodal) stimulation over left DLPFC with greater confidence.

tDCS over left DLPFC did not affect distractor inhibition processes, as indexed by $\mathrm{T} 2$ priming. However, we found that $\mathrm{T} 2$ priming may not provide a reliable measure of distractor inhibition ability at the individual participant level, as no significant correlation was observed between T2 priming in the baseline blocks of the two sessions. Dux and Marois (2008) and Slagter and Georgopoulou (2013) reported a relationship between this measure of distractor inhibition (T2/T1 at Lag 4 in prime-present vs. prime-absent trials) and recovery from the $\mathrm{AB}$ (i.e., T2/T1 at Lag 10 - Lag 4 in prime-absent trials). However, note that this correlation may be spurious because these two indices share a variable (i.e., T2/T1 at Lag 4 in no-prime trials). Nonindependent variables such as $\mathrm{AB}$ recovery and $\mathrm{T} 2$ priming can be expected to show an average correlation of .50 (in case of equal variances), even in the complete absence of a true relationship (see Elliott \& Giesbrecht, 2015). Moreover, Slagter and Georgopoulou (2013) did not observe a relationship between distractor inhibition and $\mathrm{AB}$ magnitude (i.e., T2/T1 at Lag 10 - Lag 2). Together with the here observed low test-retest reliability of the T2 priming measure and the possibly spurious correlation between $\mathrm{T} 2$ priming and $\mathrm{AB}$ recovery, this casts doubt on the previously reported relationship between distractor inhibition and $\mathrm{AB}$ recovery. Nevertheless, findings from several studies using independent measures of distractor inhibition do confirm a role for individual differences in distractor inhibition ability in the $\mathrm{AB}$ (Arnell \& Stubitz, 2010; Martens \& Valchev, 2009), and it is clear that, at least, the distractor immediately following T1 plays a critical role in the $\mathrm{AB}$ (Olivers \& Meeter, 2008; Di Lollo et al., 2005; Chun \& Potter, 1995). Here, the priming distractor was presented at Lag 2, so the priming effect may have also been weakened because of strong suppression triggered by the Lag 1 distractor and/or resource depletion because of $\mathrm{T} 1$ processing.

To conclude, atDCS over left DLPFC modulated the $A B$ as a function of baseline $\mathrm{AB}$ magnitude, supporting a modulating role for $\mathrm{pFC}$ in the $\mathrm{AB}$. This baseline-dependent effect may be explained by an inverted-U-shaped relationship between prefrontal excitability and $\mathrm{AB}$ magnitude and emphasizes the need to take baseline levels of performance into account when studying the effects of tDCS.

\section{UNCITED REFERENCE}

Sergent, Baillet, \& Dehaene, 2005 


\section{Acknowledgments}

This work was supported by a Marie Curie IRG grant and a VIDI grant by the Netherlands Scientific Organization for Research to H. A. S. We thank Chris Olivers for helpful comments on an earlier draft of this manuscript.

Reprint requests should be sent to Heleen A. Slagter, Department of Psychology, Amsterdam Brain and Cognition, University of Amsterdam, Weesperplein 4, Amsterdam, Netherlands, 1018 XA, or via e-mail: h.a.slagter@uva.nl.

\section{REFERENCES}

Arend, I., Johnston, S., \& Shapiro, K. (2006). Task-irrelevant visual motion and flicker attenuate the attentional blink. Psychonomic Bulletin \& Review, 13, 600-607.

Arnell, K. M., \& Stubitz, S. M. (2010). Attentional blink magnitude is predicted by the ability to keep irrelevant material out of working memory. Psychological Research, 74, 457-467.

Arnsten, A. F. (1998). Catecholamine modulation of prefrontal cortical cognitive function. Trends in Cognitive Sciences, 2, 436-447.

Benwell, C. S., Learmonth, G., Miniussi, C., Harvey, M., \& Thut, G. (2015). Non-linear effects of transcranial direct current stimulation as a function of individual baseline performance: Evidence from biparietal tDCS influence on lateralized attention bias. Cortex, 69, 152-165.

Bertolino, A., Blasi, G., Latorre, V., Rubino, V., Rampino, A., Sinibaldi, L., et al. (2006). Additive effects of genetic variation in dopamine regulating genes on working memory cortical activity in human brain. Journal of Neuroscience, 26, 3918-3922.

Bikson, M., \& Datta, A. (2012). Guidelines for precise and accurate computational models of tDCS. Brain Stimulation 5, 430-431.

Bindman, L. J., Lippold, O. C., \& Redfearn, J. W. (1962). Long-lasting changes in the level of the electrical activity of the cerebral cortex produced bypolarizing currents. Nature, 196, 584-585.

Brunoni, A. R., Amadera, J., Berbel, B., Volz, M. S., Rizzerio, B. G., \& Fregni, F. (2011). A systematic review on reporting and assessment of adverse effects associated with transcranial direct current stimulation. International Journal of Neuropsychopharmacology, 14, 1133-1145.

Chun, M. M., \& Potter, M. C. (1995). A two-stage model for multiple target detection in rapid serial visual presentation. Journal of Experimental Psychology: Human Perception and Performance, 21, 109-127.

Cools, R., \& D'Esposito, M. (2011). Inverted-U-shaped dopamine actions on human working memory and cognitive control. Biological Psychiatry, 69, e113-e125.

Dale, G., Dux, P. E., \& Arnell, K. M. (2013). Individual differences within and across attentional blink tasks revisited. Attention, Perception, \& Psychophysics, 75, 456-467.

DaSilva, A. F., Volz, M. S., Bikson, M., \& Fregni, F. (2011). Electrode positioning and montage in transcranial direct current stimulation. Journal of Visualized Experiments.

Datta, A., Truong, D., Minhas, P., Parra, L. C., \& Bikson, M. (2012). Inter-individual variation during transcranial direct current stimulation and normalization of dose using MRIderived computational models. Frontiers in Psychiatry, 3, 91.

Di Lollo, V., Kawahara, J., Ghorashi, S. M. S., \& Enns, J. T. (2005). The attentional blink: Resource depletion or temporary loss of control? Psychological Research, 69, 191-200.
Dickinson, D., \& Elvevåg, B. (2009). Genes, cognition and brain through a COMT lens. Neuroscience, 164, 72-87.

Dux, P. E., \& Marois, R. (2008). Distractor inhibition predicts individual differences in the attentional blink. PLoS One, 3, e3330

Dux, P. E., \& Marois, R. (2009). The attentional blink: A review of data and theory. Attention, Perception, E Psychophysics, 71, 1683-1700.

Elliott, J. C., \& Giesbrecht, B. (2015). Distractor suppression when attention fails: Behavioral evidence for a flexible selective attention mechanism. PLoS One, 10, e0126203. http://dx.doi.org/10.1371/journal.pone.0126203.

Feredoes, E., Heinen, K., Weiskopf, N., Ruff, C., \& Driver, J. (2011). Causal evidence for frontal involvement in memory target maintenance by posterior brain areas during distracter interference of visual working memory. Proceedings of the National Academy of Sciences, U.S.A., 108, 17510-17515.

Fregni, F., Boggio, P. S., Nitsche, M., Bermpohl, F., Antal, A., Feredoes, E., et al. (2005). Anodal transcranial direct current stimulation of prefrontal cortex enhances working memory. Experimental Brain Research, 166, 23-30.

Gross, J., Schmitz, F., Schnitzler, I., Kessler, K., Shapiro, K., Hommel, B., et al. (2004). Modulation of long-range neural synchrony reflects temporal limitations of visual attention in humans. Proceedings of the National Academy of Sciences, U.S.A., 101, 13050-13055.

Hommel, B., Kessler, K., Schmitz, F., Gross, J., Akyürek, E., Shapiro, K., et al. (2006). How the brain blinks: Towards a neurocognitive model of the attentional blink. Psychological Research, 70, 425-435.

Horvath, J. C., Forte, J. D., \& Carter, O. (2014). Evidence that transcranial direct current stimulation (tDCS) generates littleto-no reliable neurophysiologic effect beyond MEP amplitude modulation in healthy human subjects: A systematic review. Neuropsychologia.

Jefferies, L. N., Smilek, D., Eich, E., \& Enns, J. T. (2008). Emotional valence and arousal interact in attentional control. Psychological Science, 19, 290-295.

Jin, P. (1992). Toward a reconceptualization of the law of initial value. Psychological Bulletin, 111, 176-184.

Kawahara, J. I., Enns, J. T., \& Di Lollo, V. (2006). The attentional blink is not a unitary phenomenon. Psychological Research, 70, 405-413.

Kawahara, J. I., Kumada, T., \& Di Lollo, V. (2006). The attentional blink is governed by a temporary loss of control. Psychonomic Bulletin E Review, 13, 886-890.

Keeser, D., Padberg, F., Reisinger, E., Pogarell, O., Kirsch, V., Palm, U., et al. (2011). Prefrontal direct current stimulation modulates resting EEG and event-related potentials in healthy subjects: A standardized low resolution tomography (sLORETA) study. Neuroimage, 55, 644-657.

Kim, J.-H., Kim, D.-W., Chang, W. H., Kim, Y.-H., Kim, K., \& Im, C.-H. (2014). Inconsistent outcomes of transcranial direct current stimulation may originate from anatomical differences among individuals: Electric field simulation using individual MRI data. Neuroscience Letters, 564, 6-10.

Kranczioch, C., Debener, S., Schwarzbach, J., Goebel, R., \& Engel, A. K. (2005). Neural correlates of conscious perception in the attentional blink. Neuroimage, 24, 704-714.

Krause, B., \& Cohen Kadosh, R. (2014). Not all brains are created equal: The relevance of individual differences in responsiveness to transcranial electrical stimulation. Frontiers in Systems Neuroscience, 8, 25.

Krause, B., Márquez-Ruiz, J., \& Cohen Kadosh, R. (2013). The effect of transcranial direct current stimulation: A role for cortical excitation/inhibition balance? Frontiers in Human Neuroscience, 7, 602. 
Kuo, M.-F., Paulus, W., \& Nitsche, M. A. (2008). Boosting focallyinduced brain plasticity by dopamine. Cerebral Cortex, 18 , 648-651.

Li, L. M., Uehara, K., \& Hanakawa, T. (2015). The contribution of interindividual factors to variability of response in transcranial direct current stimulation studies. Frontiers in Cellular Neuroscience, 9, 181.

López-Alonso, V., Cheeran, B., Río-Rodríguez, D., \& FernándezDel-Olmo, M. (2014). Inter-individual variability in response to non-invasive brain stimulation paradigms. Brain Stimulation, 7, 372-380.

Maki, W. S., Frigen, K., \& Paulson, K. (1997). Associative priming by targets and distractors during rapid serial visual presentation: Does word meaning survive the attentional blink? Journal of Experimental Psychology: Human Perception and Performance, 23, 1014-1034.

Marois, R., Chun, M. M., \& Gore, J. C. (2000). Neural correlates of the attentional blink. Neuron, 28, 299-308.

Martens, S., \& Valchev, N. (2009). Individual differences in the attentional blink. The important role of irrelevant information. Experimental Psychology, 56, 18-26.

Martens, S., \& Wyble, B. (2010). The attentional blink: Past, present, and future of a blind spot in perceptual awareness. Neuroscience and Biobehavioral Reviews, 34, 947-957.

Martens, S., Munneke, J., Smid, H., \& Johnson, A. (2006). Quick minds don't blink: Electrophysiological correlates of individual differences in attentional selection. Journal of Cognitive Neuroscience, 18, 1423-1438.

McNab, F., \& Klingberg, T. (2008). Prefrontal cortex and basal ganglia control access to working memory. Nature Neuroscience, 11, 103-107.

Myrtek, M., \& Foerster, F. (1986). The law of initial value: A rare exception. Biological Psychology, 22, 227-237.

Nieratschker, V., Kiefer, C., Giel, K., Krüger, R., \& Plewnia, C. (2015). The COMT val/met polymorphism modulates effects of tDCS on response inhibition. Brain Stimulation, 8 , 283-288.

Nieuwenstein, M. R. (2006). Top-down controlled, delayed selection in the attentional blink. Journal of Experimental Psychology: Human Perception and Performance, 32, 973.

Nieuwenstein, M. R., \& Potter, M. C. (2006). Temporal limits of selection and memory encoding a comparison of whole versus partial report in rapid serial visual presentation. Psychological Science, 17, 471-475.

Nitsche, M. A., \& Paulus, W. (2000). Excitability changes induced in the human motor cortex by weak transcranial direct current stimulation. Journal of Physiology (London), 527, 633-639.

Nitsche, M. A., \& Paulus, W. (2001). Sustained excitability elevations induced by transcranial DC motor cortex stimulation in humans. Neurology, 57, 1899-1901.

Nitsche, M. A., Cohen, L. G., Wassermann, E. M., Priori, A., Lang, N., Antal, A., et al. (2008). Transcranial direct current stimulation: State of the art 2008. Brain Stimulation, 1, 206-223.

Nitsche, M. A., Fricke, K., Henschke, U., Schlitterlau, A., Liebetanz, D., Lang, N., et al. (2003). Pharmacological modulation of cortical excitability shifts induced by transcranial direct current stimulation in humans. Journal of Physiology (London), 553, 293-301.

Nitsche, M. A., Lampe, C., Antal, A., Liebetanz, D., Lang, N., Tergau, F., et al. (2006). Dopaminergic modulation of long-lasting direct current-induced cortical excitability changes in the human motor cortex. European Journal of Neuroscience, 23, 1651-1657.
Nitsche, M. A., Monte-Silva, K., Kuo, M.-F., \& Paulus, W. (2010). Dopaminergic impact on cortical excitability in humans. Reviews in the Neurosciences, 21, 289-298.

Olivers, C. N. L., \& Meeter, M. (2008). A boost and bounce theory of temporal attention. Psychological Review, 115, 836-863.

Olivers, C. N. L., \& Nieuwenhuis, S. (2006). The beneficial effects of additional task load, positive affect, and instruction on the attentional blink. Journal of Experimental Psychology: Human Perception and Performance, 32, 364-379.

Olivers, C. N., Van Der Stigchel, S., \& Hulleman, J. (2007). Spreading the sparing: Against a limited-capacity account of the attentional blink. Psychological Research, 71, 126-139.

Plewnia, C., Zwissler, B., Längst, I., Maurer, B., Giel, K., \& Krüger, R. (2013). Effects of transcranial direct current stimulation (tDCS) on executive functions: Influence of COMT Val/Met polymorphism. Cortex, 49, 1801-1807.

Poreisz, C., Boros, K., Antal, A., \& Paulus, W. (2007). Safety aspects of transcranial direct current stimulation concerning healthy subjects and patients. Brain Research Bulletin, 72, 208-214.

Postle, B. R. (2006). Working memory as an emergent property of the mind and brain. Neuroscience, 139, 23-38.

Raghunathan, T. E., Rosenthal, R., \& Rubin, D. B. (1996). Comparing correlated but nonoverlapping correlations. Psychological Methods, 1, 178-183.

Raymond, J. E., Shapiro, K. L., \& Arnell, K. M. (1992). Temporary suppression of visual processing in an RSVP task: An attentional blink? Journal of Experimental Psychology: Human Perception and Performance, 18, 849-860.

Sadleir, R. J., Vannorsdall, T. D., Schretlen, D. J., \& Gordon, B. (2010). Transcranial direct current stimulation (tDCS) in a realistic head model. Neuroimage, 51, 1310-1318.

Sergent, C., Baillet, S., \& Dehaene, S. (2005). Timing of the brain events underlying access to consciousness during the attentional blink. Nature Neuroscience, 8, 1391-1400.

Shapiro, K. L., Raymond, J. E., \& Arnell, K. M. (1994). Attention to visual pattern information produces the attentional blink in rapid serial visual presentation. Journal of Experimental Psychology: Human Perception and Performance, 20, 357-371.

Shapiro, K. L., Raymond, J. E., \& Arnell, K. M. (1997). The attentional blink. Trends in Cognitive Sciences, 1 291-296.

Slagter, H. A., \& Georgopoulou, K. (2013). Distractor inhibition predicts individual differences in recovery from the attentional blink. PLoS One, 8, e64681.

Slagter, H. A., Johnstone, T., Beets, I. A. M., \& Davidson, R. J. (2010). Neural competition for conscious representation across time: An fMRI study. PLoS One, 5, e10556.

Teo, F., Hoy, K. E., Daskalakis, Z. J., \& Fitzgerald, P. B. (2011). Investigating the role of current strength in tDCS modulation of working memory performance in healthy controls. Frontiers in Psychiatry, 2, 45.

Thayer, R. E. (1978). Factor analytic and reliability studies on the Activation-Deactivation Adjective Check List. Psychological Reports, 42, 747-756.

Tu, Y.-K., \& Gilthorpe, M. S. (2007). Revisiting the relation between change and initial value: A review and evaluation. Statistics in Medicine, 26, 443-457.

Williams, G. V., \& Goldman-Rakic, P. S. (1995). Modulation of memory fields by dopamine D1 receptors in prefrontal cortex. Nature, 376, 572-575.

Zahrt, J., Taylor, J. R., Mathew, R. G., \& Arnsten, A. F. (1997). Supranormal stimulation of D1 dopamine receptors in the rodent prefrontal cortex impairs spatial working memory performance. Journal of Neuroscience, 17, 8528-8535. 


\section{AUTHOR QUERIES}

\section{AUTHOR PLEASE ANSWER ALL QUERIES}

During the preparation of your manuscript, the questions listed below arose. Kindly supply the necessary information.

1. Committee on Methods of Clinical Examination in Electroencephalography, 1957 was cited in the body but not in the reference list. Please check.

2. Please provide manufacturer name and location for this product name (Presentation software).

3. Please provide manufacturer name and location for this product name (Microsoft operating system $\mathrm{XP})$.

4. Please insert citation of this reference in the body: Sergent, Baillet, \& Dehaene, 2005.

5. Please update details of this reference (DaSilva et al., 2011).

6. Please update details of this reference (Horvath et al., 2014).

\section{END OF ALL QUERIES}

